\title{
Analysis of nocturnal desaturation waveforms using algorithms in patients with idiopathic pulmonary fibrosis
}

\author{
Yuichiro Yasuda ${ }^{1}$ Tatsuya Nagano ${ }^{1}$ (D) Shintaro Izumi ${ }^{2} \cdot$ Mina Yasuda $^{3} \cdot$ Kosuke Tsuruno $^{3} \cdot$ Kazunori Tobino $^{3}$. \\ Kyosuke Nakata ${ }^{1,4} \cdot$ Kayoko Okamura $^{5} \cdot$ Teruaki Nishiuma $^{6} \cdot$ Kiyonobu Takatsuki $^{7} \cdot$ Yasuhiro Funada $^{8}$. \\ Hisashi Ohnishi $^{5} \cdot$ Masatsugu Yamamoto $^{1} \cdot$ Yoshihiro Nishimura $^{1} \cdot$ Kazuyuki Kobayashi $^{1}$
}

Received: 25 March 2021 / Revised: 20 July 2021 / Accepted: 28 July 2021 / Published online: 21 August 2021

(c) The Author(s) 2021

\begin{abstract}
Purpose Sleep-disordered breathing is recognized as a comorbidity in patients with idiopathic pulmonary fibrosis (IPF). Among them, nocturnal hypoxemia has been reported to be associated with poor prognosis and disease progression. We developed a diagnostic algorithm to classify nocturnal desaturation from percutaneous oxygen saturation $\left(\mathrm{SpO}_{2}\right)$ waveform patterns: sustained pattern, periodic pattern, and intermittent pattern. We then investigated the prevalence of nocturnal desaturation and the association between the waveform patterns of nocturnal desaturation and clinical findings of patients with IPF. Methods We prospectively enrolled patients with IPF from seven general hospitals between April 2017 and March 2020 and measured nocturnal $\mathrm{SpO}_{2}$ and nasal airflow by using a home sleep apnea test. An algorithm was used to classify the types of nocturnal desaturation. We evaluated the association between sleep or clinical parameters and each waveform pattern of nocturnal desaturation.

Results Among 60 patients (47 men) who met the eligibility criteria, there were 3 cases with the sustained pattern, 49 cases with the periodic pattern, and 41 cases with the intermittent pattern. Lowest $\mathrm{SpO}_{2}$ during sleep and total sleep time spent with $\mathrm{SpO}_{2}<90 \%$ were associated with the sustained pattern, and apnea-hypopnea index was associated with the intermittent pattern. Conclusion We demonstrated the prevalence of each waveform and association between each waveform and sleep parameters in patients with IPF. This classification algorithm may be useful to predict the degree of hypoxemia or the complication of obstructive sleep apnea.
\end{abstract}

Keywords Home sleep apnea test $\cdot$ Idiopathic pulmonary fibrosis $\cdot$ Nocturnal hypoxemia $\cdot$ Percutaneous oxygen saturation

$\begin{array}{ll}\text { Abbreviations } \\ \text { 6MWT } & \text { The six-minute walk test } \\ \mathrm{AHI} & \text { Apnea-hypopnea index } \\ \mathrm{DLCO} & \text { Diffusing capacity for carbon monoxide } \\ \text { FVC } & \text { Forced vital capacity } \\ \mathrm{HOT} & \text { Home oxygen therapy } \\ \mathrm{ILD} & \text { Interstitial lung disease } \\ \mathrm{IPF} & \text { Idiopathic pulmonary fibrosis } \\ \mathrm{OSA} & \text { Obstructive sleep apnea } \\ \mathrm{PaO}_{2} & \text { Partial pressure of oxygen in arterial blood } \\ \mathrm{PFT} & \text { Pulmonary function test } \\ \mathrm{PSG} & \text { Polysomnography } \\ \mathrm{SpO}_{2} & \text { Percutaneous oxygen saturation }\end{array}$

Tatsuya Nagano

tnagano@med.kobe-u.ac.jp

Extended author information available on the last page of the article
TRPG Transtricuspid pressure gradient

TST90 Total sleep time spent with $\mathrm{SpO}_{2}<90$

\section{Introduction}

Idiopathic pulmonary fibrosis (IPF) is defined as a specific form of chronic, progressive, fibrosing interstitial pneumonia of unknown cause [1]. The median survival time after diagnosis has been reported to be approximately 35 months [2]. The management of various comorbidities with IPF is a clinically important issue. Obstructive sleep apnea (OSA) is one of the comorbidities [3]. However, less research has been focused on changes in oxygenation and breathing patterns in IPF during sleep compared to daytime oxygenation. Including OSA, nocturnal hypoxemia is reported to be observed occasionally in IPF patients [4]. Troy and colleagues showed that nocturnal hypoxemia is associated with poor prognosis and 
may contribute to the development of pulmonary hypertension in interstitial lung disease (ILD) patients [5]. Also, the presence of OSA with nocturnal hypoxemia has been reportedly associated with poor prognosis and disease progression in IPF patients [6]. Pulmonary function tended to be worse in the group with both OSA and nocturnal hypoxemia than in the group without sleep breathing disorder and the group with OSA only. These observations suggest the association between progression in nocturnal disorder and the disease stage of IPF. The recognition of nocturnal hypoxemia in IPF patients might shed light not only on diagnosing such complications but also on associating the disease progression itself of IPF.

In our previous study, we monitored nocturnal percutaneous oxygen saturation $\left(\mathrm{SpO}_{2}\right)$ level in the patients with chronic respiratory disease that needed home oxygen therapy (HOT) [7]. This study confirmed that nocturnal desaturation occurred even in the patients in whom HOT improved daytime hypoxemia. Also, the waveform pattern differed greatly among $\mathrm{SpO}_{2}$ waveform patterns. Then, we developed a diagnostic algorithm according to the pathophysiological condition for $\mathrm{SpO}_{2}$ waveform patterns: sustained pattern, periodic pattern, and intermittent pattern (examples of the waveforms are presented in Fig. 1) [7], on the basis of the respiratory pathophysiology under HOT, including the duration of desaturation and frequent repetition of drop and recover in $\mathrm{SpO}_{2}$. Although the algorithm was developed based on the oxygen therapy, we sought that the pathophysiology on the desaturation fluctuation could be applied to other respiratory diseases even without oxygen therapy. Since it has not yet been investigated in individual respiratory diseases without oxygen therapy, this study focused on IPF. We aimed to investigate the prevalence of nocturnal desaturation in IPF and to determine the association between the waveform patterns of nocturnal desaturation and clinical findings.

\section{Patients and methods}

\section{Patients}

This multicenter prospective study was performed between April 2017 and March 2020 at seven general hospitals in

(A)

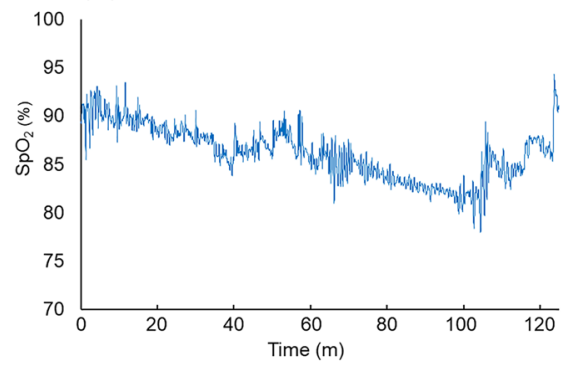

(B)

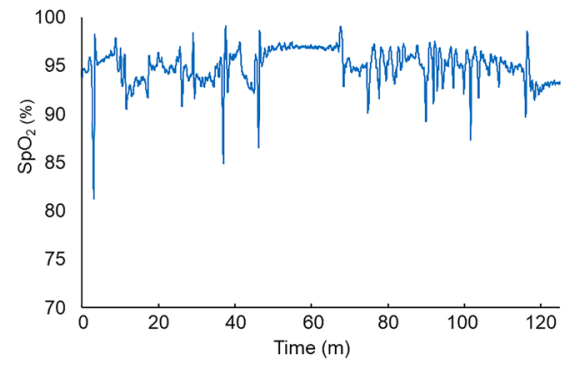

64 patients were enrolled

1 patients could not be evaluate overnight $\mathrm{SpO}_{2}$

63 patients were evaluated overnight $\mathrm{SpO}_{2}$

3 patients were unable to collect data

60 patients were analyzed

Fig. 2 Flow chart of patient recruitment process

Japan, namely, Kobe University Hospital, Iizuka Hospital, Kasai City Hospital, Kakogawa Central City Hospital, Kitaharima Medical Center, Akashi Medical Center, and Takatsuki General Hospital. We enrolled 64 outpatients with IPF who met the following criteria: were aged 20 years or older; were not undergoing HOT; had daytime $\mathrm{SpO}_{2}>88 \%$ or partial pressure of oxygen in arterial blood $\left(\mathrm{PaO}_{2}\right)>55$ torr. IPF was diagnosed based on international guidelines [1]. A flowchart showing the patient recruitment process is shown in Fig. 2. This study was approved by the institutional review board at Kobe University Hospital (approval number: 170017).

\section{Home sleep apnea test}

$\mathrm{SpO}_{2}$ and nasal airflow were recorded continuously using a SAS-2100 device (Nihon Kohden Corporation, Tokyo, Japan). Apnea-hypopnea index (AHI, average apnea and hypopnea per hour of sleep time), total sleep time, and lowest $\mathrm{SpO}_{2}$ were recorded. Apnea is defined as a reduction in signal amplitude to $10 \%$ of baseline for at least $10 \mathrm{~s}$. Hypopnea is defined as a reduction in signal amplitude to $70 \%$ of baseline and $\geq 3 \%$ oxygen desaturation for at least $10 \mathrm{~s}$. Patients with a total sleep time of less than $4 \mathrm{~h}$ were excluded from the analysis.

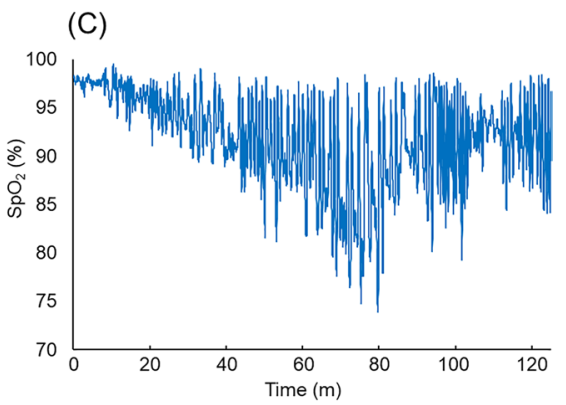

Fig. 1 Examples of waveforms of nocturnal desaturation. Sustained pattern (A), periodic pattern $(\mathbf{B})$, and intermittent pattern $(\mathbf{C})$ 


\section{Pulmonary function test}

Pulmonary function test (PFT) and the measurement of diffusing capacity for carbon monoxide (DLCO) were performed according to the international recommendations $[8$, 9] within 3 months of enrollment in each hospital. Japanese local reference values were used for the predicted values of PFT [10].

\section{Six-minute walk test}

The six-minute walk test (6MWT) was performed in all patients by trained technicians. The 6-min walk distance and lowest $\mathrm{SpO}_{2}$ value were recorded.

\section{Other clinical data}

Gender, height, weight, comorbidities, smoking history, medications, daytime $\mathrm{SpO}_{2}$ and $\mathrm{PaO}_{2}$ at rest, echocardiography-measured ejection fraction and transtricuspid pressure gradient (TRPG), subjective breathlessness scale (modified medical research council), and laboratory data including sialylated carbohydrate antigen Krebs von den Lungen-6 and surfactant protein-D were measured or extracted from patients' medical record.

\section{Severity of IPF}

IPF patients were classified as stage $\mathrm{I}\left(\mathrm{PaO}_{2}>80 \mathrm{mmHg}\right.$ at rest), stage II (70-79 $\mathrm{mmHg})$, stage III $(60-69 \mathrm{mmHg})$, or stage IV $(<59 \mathrm{mmHg})$ based on the severity stage used clinically in Japan. Among patients with stage II or III, the severity is increased by one stage if the lowest $\mathrm{SpO}_{2}$ is less than $90 \%$ during a 6 MWT [11].

\section{Classification algorithm of nocturnal desaturation}

Desaturation was defined as the decrease in $\mathrm{SpO}_{2}$ by more than $3 \%$ from baseline. The cases with nocturnal desaturation were diagnosed based on the reported algorithm into three classifications, namely, sustained pattern, periodic pattern, and intermittent pattern [7]. Briefly, when one or more drop events longer than $655 \mathrm{~s}$ occur, the measured data were labeled as the sustained pattern. When the drop event between 30 and $655 \mathrm{~s}$ occurred more than twice during the measurement period, the pattern was labeled as the periodic pattern. We defined the third pattern for characteristic frequency pattern seen in OSA, intermittent pattern where the drop and recovery in $\mathrm{SpO}_{2}$ was repeated with a cycle of several minutes. In addition, the time-frequency analysis using discrete Fourier transform was conducted for $\mathrm{SpO}_{2}$ data (Figure S1). The window length was set to $600 \mathrm{~s}$ and the maximum spectral power between $0.7 / 60$ and $1.5 / 60 \mathrm{~Hz}$ is calculated. The intermittent pattern was set if the maximum spectral power was larger than 2.0 and this state occurred for more than $1300 \mathrm{~s}$ per hour. Each pattern of desaturation can overlap in a patient because of the distinct algorithm which is not exclusive of the other patterns. The flowchart of the classification for nocturnal desaturation is shown in Fig. 3.

\section{Sample size}

According to previous reports, $37 \%$ of patients with ILD showed nocturnal hypoxemia [12]. The proportions of cases assigned to stages I, II, III, and IV were $43 \%, 13 \%, 22 \%$, and $22 \%$, respectively [13]. Here, we hypothesized that the prevalence of nocturnal desaturation depends on the IPF severity. Since there was a difference in the prognosis between the cases in stages I/II and III/IV, we divided the current patients into two groups. In our hypothesis, we estimated that the prevalence rates of nocturnal hypoxemia in stage I/II and stage III/ IV are $20 \%$ and $60 \%$, respectively. We estimated that 31 and 24 patients were needed in the groups with a two-sided alpha value of 0.05 and a statistical power value of $80 \%$.

\section{Statistical analysis}

Fisher's exact test was used for between-group comparisons of the categorical variables. As exploratory analyses, logistic regression analysis and multiple regression analysis were conducted to determine clinical parameters associated with each waveform classification. All $P$ values reported are 2 -sided, and $P$ values less than 0.05 were considered significant. All of the statistical analyses were performed with EZR (Saitama Medical Center, Jichi Medical University), which is a graphical user interface for the R software program (The R Foundation for Statistical Computing, version 2.13.0) [14]. It is a modified version of $\mathrm{R}$ commander (version 1.27) that includes statistical functions that are used in biostatistics.

\section{Results}

\section{Patient characteristic}

Of 60 patients ( 47 men) who met the eligibility criteria (Table 1), the number of patients with stages I/II and III/IV was 43 (72\%) and $17(28 \%)$, respectively. The mean forced vital capacity (FVC) and DLCO were $81 \%$ and $61 \%$ predicted, respectively. Thirty-two patients underwent TTE, but no patients had elevated TRPG; therefore, we did not analyze the association of pulmonary hypertension in this study. 

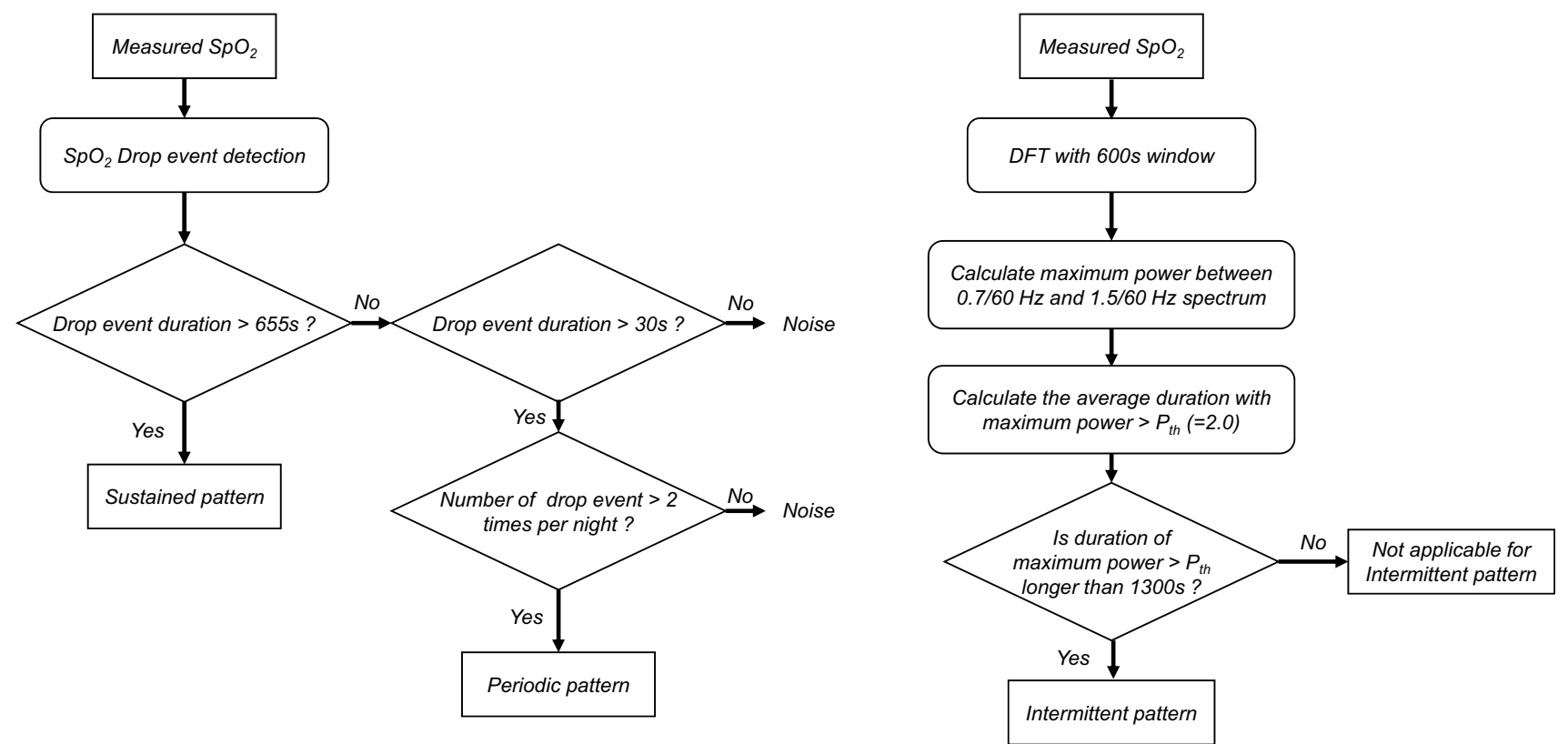

Fig. 3 Flowchart of nocturnal desaturation pattern judgment

\section{Nocturnal desaturation and its classification}

Of 60 cases, $52(87 \%)$ corresponded with any of the nocturnal desaturation patterns. The sleep characteristics are summarized in Table 2. The number of patients showing nocturnal desaturation was $3(5 \%), 49(82 \%)$, and $41(68 \%)$ with sustained pattern, periodic pattern, and intermittent pattern, respectively. There were eight patients who did not meet each pattern criteria. A Venn diagram for the waveform pattern is shown in Fig. 4. For the IPF severity, in severity stage I/II (43 patients), there were 2 patients $(4 \%), 35$ patients $(81 \%)$, and 29 patients $(67 \%)$, in the sustained, periodic, and intermittent patterns, respectively. Also, in severity stage III/IV (17 patients), there were 1 patient $(6 \%)$, 14 patients $(82 \%)$, and 12 patients $(71 \%)$, respectively. There was no significant difference between stages I/II and III/IV regarding the waveform patterns. The clinical and sleep characteristics with sustained pattern are described in Table S1. Only 3 patients showed desaturation with the sustained pattern. The DLCO $\%$ predicted decreased in all three cases. Moreover, one of the three patients showed an AHI of 6.3. Separately, we analyzed 10 healthy subjects using the algorithm for the waveform classifications. All healthy subjects met none of the classifications for nocturnal desaturation (Table S2).

\section{Association between nocturnal oxygen saturation and the waveform patterns}

As exploratory analyses to assess the association between the classifications by the algorithm and nocturnal oxygen saturation, we performed the logistic regression analyses for modeling each waveform pattern. AHI, lowest $\mathrm{SpO}_{2}$, and TST90 were included as predictor variables. Lowest $\mathrm{SpO}_{2}$ and TST90 were associated with the sustained pattern (lowest $\mathrm{SpO}_{2}$, odds ratio (OR): $0.796,95 \%$ confidence interval (CI): $0.637-0.994, P$ value $=0.04$; TST90, OR: $1.1,95 \%$ CI: $1.01-1.18, P$ value $=0.02)$. AHI was associated with the intermittent pattern (AHI, OR: $1.54,95 \% \mathrm{CI}: 1.16-2.06, P$ value $<0.005)$. They were not significantly associated with the periodic pattern.

\section{Association between daytime clinical parameters and the waveform patterns}

Next, in order to analyze the association between the daytime clinical parameters and each waveform pattern, we conducted the logistic regression analysis and multivariable regression analysis. No significant associations were found between lower DLCO ( $<60 \%$ predicted) and any of the waveform patterns. FVC (\% predicted) tended to be higher, although not significantly, in the intermittent group compared with the others $(P$ value $=0.07$ )

\section{Discussion}

In the present study, we prospectively analyzed the waveform patterns in nocturnal desaturation in patients with IPF. There was no significant difference between stages I/II and III/IV regarding the waveform patterns. Persistent nocturnal hypoxemia was less than a priori 
Table 1 Patients' characteristics $(n=60)$

\begin{tabular}{|c|c|}
\hline \multicolumn{2}{|l|}{ Sex } \\
\hline Male/female & $47 / 13$ \\
\hline Age, yr & $72.5 \pm 8.6$ \\
\hline BMI & $24.1 \pm 3.2$ \\
\hline \multicolumn{2}{|l|}{ Smoking status, $n(\%)$} \\
\hline Never/former/current & $16(26.7) / 39(65) / 5(8.3)$ \\
\hline Daytime $\mathrm{PaO}_{2}(\mathrm{mmHg})$ & $83.9 \pm 11.9$ \\
\hline Daytime $\mathrm{SpO}_{2}(\%)$ & $96.5 \pm 1.4$ \\
\hline \multicolumn{2}{|l|}{ IPF severity, $n(\%)$} \\
\hline I/II/III/IV & $36(60) / 7(11.7) / 12(20) / 5(8.3)$ \\
\hline \multicolumn{2}{|l|}{ mMRC, $n(\%)$} \\
\hline $0 / 1 / 2 / 3 / 4$ & $16(26.7) / 21(35) / 13(21.7) / 10(16.7) / 0(0)$ \\
\hline \multicolumn{2}{|l|}{ Pulmonary function test } \\
\hline $\mathrm{VC}, \%$ predicted & $79.5 \pm 17.3$ \\
\hline FVC, $\%$ predicted & $81 \pm 17.7$ \\
\hline FEV1, \% predicted & $84 \pm 19.4$ \\
\hline DLCO, $\%$ predicted & $60.8 \pm 16.9$ \\
\hline \multicolumn{2}{|l|}{$6 \mathrm{MWT}$} \\
\hline Distance (m) & $372 \pm 120$ \\
\hline Lowest $\mathrm{SpO}_{2}(\%)$ & $90 \pm 4.5$ \\
\hline KL-6 (U/ml) & $937 \pm 673$ \\
\hline SP-D (ng/ml) & $223 \pm 147$ \\
\hline TRPG $>40 \mathrm{mmHg}$ on TTE, n/total & $0 / 32$ \\
\hline \multicolumn{2}{|l|}{ Past medical history, n (\%) } \\
\hline Hypertension & $28(46.7)$ \\
\hline Diabetes mellitus & $19(31.7)$ \\
\hline Gastroesophageal reflux disease & $1(1.6)$ \\
\hline Ischemic heart disease & $5(8.3)$ \\
\hline Chronic obstructive pulmonary disease & $4(6.7)$ \\
\hline Lung cancer & $5(8.3)$ \\
\hline Depression & $0(0)$ \\
\hline Neuromuscular disorder & $0(0)$ \\
\hline \multicolumn{2}{|l|}{ Medication, $n(\%)$} \\
\hline Oral corticosteroids & $3(5)$ \\
\hline Sleeping medicine & $2(3.3)$ \\
\hline Anti-fibrotic drug & $23(38.3)$ \\
\hline
\end{tabular}

The data are expressed as the number or mean $\pm \mathrm{SD}$

$B M I$, body mass index, $I P F$, idiopathic pulmonary fibrosis, $V C$, vital capacity, $F V C$, forced vital capacity, $F E V 1$, forced expiratory volume in one second, DLCO: diffusing capacity for carbon monoxide, $6 M W T$, six-minute walk test, TRPG, transtricuspid pressure gradient, $T T E$, transthoracic echocardiography estimation, as the persistent hypoxemia was observed less in the IPF patients without HOT than in the patients with HOT as reported [7]. Focusing on the $\mathrm{SpO}_{2}$ fluctuation at night, however, we did observe nocturnal desaturation events in most of the IPF patients. These results suggest a subclinical disordered respiration even in IPF patients without hypoxemia.

Exploratory analysis of waveform classification and sleep parameters suggested that the degree and duration of nocturnal desaturation (lowest $\mathrm{SpO}_{2}$ and TST90) was associated with the sustained pattern. Three patients with the sustained 
Table 2 Sleep characteristics

\begin{tabular}{ll}
\hline Total sleep time (min) & $455 \pm 90$ \\
AHI & $19.3 \pm 13.8$ \\
Lowest $\mathrm{SpO}_{2}(\%)$ & $81.5 \pm 6.5$ \\
TST90 $(\%)$ & $1.3(0.1-3.1)$ \\
Classification of nocturnal desaturation, $n(\%)$ & \\
$\quad$ Sustained & $3(5)$ \\
$\quad$ Periodic & $49(82)$ \\
$\quad$ Intermittent & $41(68)$ \\
$\quad$ Not applicable & $8(13)$ \\
\hline
\end{tabular}

The data are expressed as the number or mean \pm SD or median (interquartile range)

AHI, apnea-hypopnea index, TST90, total sleep time with $\mathrm{SpO}_{2}<90 \%$

pattern had decreased DLCO; however, preserved AHI despite sustained desaturation in one of such patients might shed light on the difficulty in assessing nocturnal hypoxemia solely by AHI. A recent study on nocturnal hypoxemia in ILD reported that TST90 correlated significantly with several markers of ILD disease severity including oxygenation and DLCO [5]. In addition, it may be necessary to verify whether the waveform pattern changes when continuous oxygen therapy is given to patients with sustained pattern.

Together with the algorithm setting that the sustained pattern is defined as desaturation over $10 \mathrm{~min}$, these findings raise speculation that the sustained pattern might appear in IPF patients with relatively progressive disease, but sample size limited the further analysis in the current study. Future studies to optimize the algorithm including cut-off value of

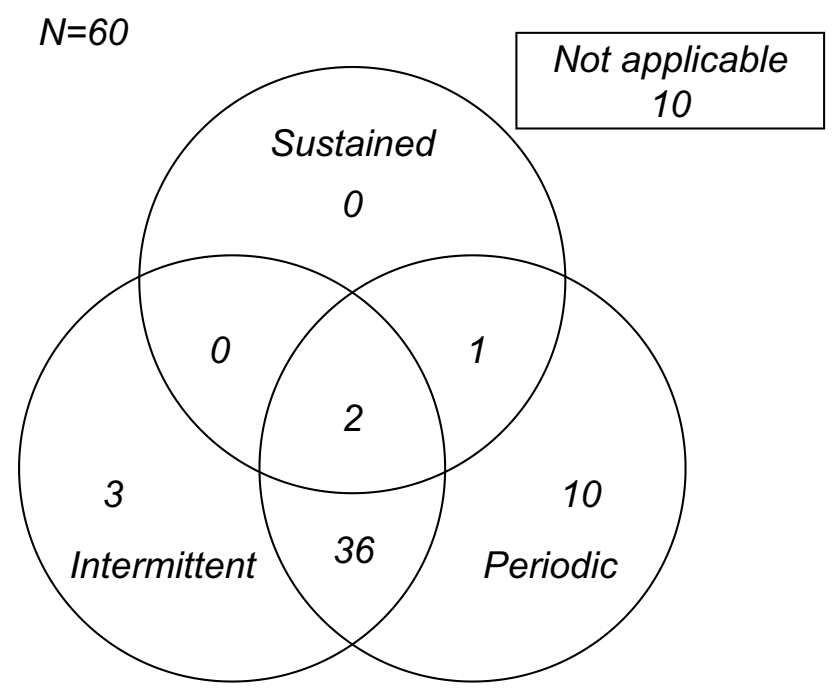

Fig. 4 Venn diagram of each waveform in patients with idiopathic pulmonary fibrosis duration might lead to clarify the pathophysiological link to the sustained pattern and disease progression.

Our results showing that the intermittent pattern was associated with AHI suggested that this classification may predict the degree of hypoxemia or the complication of OSA. None of the sleep parameters showed a significant association with the periodic pattern.

Since the intermittent pattern correlates with AHI, this pattern seems to reflect sleep apnea. In the present study, intermittent pattern was found in $68 \%$, which is considered to be a similar prevalence of OSA as reported previously $[6,15]$. Given that it is necessary to recognize OSA in IPF patients, nocturnal desaturation with intermittent pattern might predict OSA or related disorder. In patients with the intermittent pattern, FVC\% predicted tended to be maintained compared to the other classifications. It suggests that the waveform can change from an intermittent to a sustained pattern as the clinical course of IPF progression. The followup observational survey tracing nocturnal desaturation pattern might reveal the natural history of sleep-related disorder in IPF.

The periodic patterns were primarily assumed in the development as the desaturation events including rapid eye movement sleep-related hypoventilation. Primary observation revealed that many patients with HOT have periodic pattern [7]. In the current study, the periodic pattern was neither specific to IPF patients nor related to daytime and sleep parameters. Hence, the clinical implication of this waveform pattern was not clear in this study. Further study using polysomnography (PSG) for waveform classification may help elucidate the pathophysiology of periodic pattern.

The SAS-2100 is a portable sleep apnea tester which is capable of measuring airflow, $\mathrm{SpO}_{2}$, and pulse rate. The total sleep time is determined from the time the machine is turned on to off. In addition, this SAS-2100 cannot detect the degree of ventilation and the level of carbon dioxide. Therefore, the limitations of the present study are that the present study lacks data on PSG or overnight transcutaneous carbon dioxide tension. Home sleep apnea test tends to underestimate AHI because the recording time is longer than sleep time. There is also the inaccuracy that hypopnea associated with arousal is measured. Regarding waveform classification, whose cut-off value was set from the examination of HOT patients, it has not been verified for application in patients with IPF.

\section{Conclusions}

We observed the prevalence of nocturnal desaturation and its classification in patients with IPF. The proof-of-concept study for the waveform algorithm showed that the patterns were related to the degree of hypoxemia or the complication 
of OSA, which can affect the prognosis in IPF. Future studies, including therapeutic interventions or PSG assessments, may reveal clinical significance of waveform classification.

Supplementary Information The online version contains supplementary material available at https://doi.org/10.1007/s11325-021-02456-3.

Acknowledgements We would like to thank all investigators who recruited patients and all the patients who participated.

Author contribution Conceptualization: Yuichiro Yasuda, Tatsuya Nagano. Data curation: Yuichiro Yasuda, Mina Yasuda, Kosuke Tsuruno, Kazunori Tobino, Kyosuke Nakata, Kayoko Okamura, Teruaki Nishiuma, Kiyonobu Takatsuki, Yasuhiro Funada, Hisashi Ohnishi. Formal analysis: Yuichiro Yasuda, Tatsuya Nagano, Masatsugu Yamamoto. Funding acquisition: Yoshihiro Nishimura. Investigation: Yuichiro Yasuda, Tatsuya Nagano, Masatsugu Yamamoto. Methodology: Yuichiro Yasuda, Tatsuya Nagano, Masatsugu Yamamoto. Project administration: Tatsuya Nagano, Yoshihiro Nishimura. Software: Shintaro Izumi. Supervision: Kazuyuki Kobayashi, Yoshihiro Nishimura. Writing - original draft: Yuichiro Yasuda. Writing—review and editing: Tatsuya Nagano, Masatsugu Yamamoto, Kazuyuki Kobayashi, Yoshihiro Nishimura.

Funding Yoshihiro Nishimura received a joint research grant from Teijin Pharma Limited.

Data availability Data are available from the corresponding author upon reasonable request.

Code availability Not applicable.

\section{Declarations}

Ethics approval This study was approved by the Institutional Review Board at Kobe University Hospital (approval number: 170017). The procedures used in this study adhere to the tenets of the Declaration of Helsinki.

Consent to participate Informed consent was obtained from all individual participants included in the study.

Consent for publication The participant has consented to the submission of the journal.

Conflict of interest Yoshihiro Nishimura received a joint research grant from Teijin Pharma Limited. Tatsuya Nagano, Shintaro Izumi and Yoshihiro Nishimura received patent royalty/licensing fee from Teijin Pharma Limited.

Open Access This article is licensed under a Creative Commons Attribution 4.0 International License, which permits use, sharing, adaptation, distribution and reproduction in any medium or format, as long as you give appropriate credit to the original author(s) and the source, provide a link to the Creative Commons licence, and indicate if changes were made. The images or other third party material in this article are included in the article's Creative Commons licence, unless indicated otherwise in a credit line to the material. If material is not included in the article's Creative Commons licence and your intended use is not permitted by statutory regulation or exceeds the permitted use, you will need to obtain permission directly from the copyright holder. To view a copy of this licence, visit http://creativecommons.org/licenses/by/4.0/.

\section{References}

1. Raghu G, Remy-Jardin M, Myers JL et al (2018) Diagnosis of idiopathic pulmonary fibrosis. An official ATS/ERS/JRS/ALAT Clinical practice guideline. Am J Respir Crit Care Med 198:e44 e68. https://doi.org/10.1164/rccm.201807-1255ST

2. Natsuizaka M, Chiba H, Kuronuma K et al (2014) Epidemiologic survey of Japanese patients with idiopathic pulmonary fibrosis and investigation of ethnic differences. Am J Respir Crit Care Med 190:773-779. https://doi.org/10.1164/rccm.201403-0566OC

3. Raghu G, Amatto VC, Behr J, Stowasser S (2015) Comorbidities in idiopathic pulmonary fibrosis patients: a systematic literature review. Eur Respir J 46:1113-1130. https://doi.org/10.1183/13993 003.02316-2014

4. Mermigkis C, Bouloukaki I, Schiza SE (2017) Sleep as a new target for improving outcomes in idiopathic pulmonary fibrosis. Chest 152:1327-1338. https://doi.org/10.1016/j.chest.2017.07. 019

5. Troy LK, Young IH, Lau EMT et al (2019) Nocturnal hypoxaemia is associated with adverse outcomes in interstitial lung disease. Respirology 24:996-1004. https://doi.org/10.1111/resp.13549

6. Bosi M, Milioli G, Fanfulla F et al (2017) OSA and prolonged oxygen desaturation during sleep are strong predictors of poor outcome in IPF. Lung 195:643-651. https://doi.org/10.1007/ s00408-017-0031-4

7. Izumi S, Nagano T, Yoshizaki A, Nishimura Y (2019) Classification algorithm for nocturnal hypoxemia using nocturnal pulse oximetry. Proc Annu Int Conf IEEE Eng Med Biol Soc EMBS 3662-3665. https://doi.org/10.1109/EMBC.2019.8856384

8. MacIntyre N, Crapo RO, Viegi G et al (2005) Standardisation of the single-breath determination of carbon monoxide uptake in the lung. Eur Respir J 26:720-735. https://doi.org/10.1183/09031936. 05.00034905

9. Miller MR, Hankinson J, Brusasco V et al (2005) Standardisation of spirometry. Eur Respir J 26:319-338. https://doi.org/10.1183/ 09031936.05.00034805

10. Kubota M, Kobayashi H, Quanjer PH et al (2014) Reference values for spirometry, including vital capacity, in Japanese adults calculated with the LMS method and compared with previous values. Respir Investig 52:242-250. https://doi.org/10.1016/j. resinv.2014.03.003

11. Homma S, Sugino K, Sakamoto S (2015) The usefulness of a disease severity staging classification system for IPF in Japan: 20 years of experience from empirical evidence to randomized control trial enrollment. Respir Investig 53:7-12. https://doi.org/ 10.1016/j.resinv.2014.08.003

12. Corte TJ, Wort SJ, Talbot S et al (2012) Elevated nocturnal desaturation index predicts mortality in interstitial lung disease. Sarcoidosis Vasc Diffus lung Dis Off J WASOG 29:41-50

13. Kondoh S, Chiba H, Nishikiori $\mathrm{H}$ et al (2016) Validation of the Japanese disease severity classification and the GAP model in Japanese patients with idiopathic pulmonary fibrosis. Respir Investig 54:327-333. https://doi.org/10.1016/j.resinv.2016.02.009

14. Kanda Y (2013) Investigation of the freely available easy-to-use software "EZR" for medical statistics. Bone Marrow Transplant 48:452-458. https://doi.org/10.1038/bmt.2012.244

15. Mermigkis C, Stagaki E, Tryfon S et al (2010) How common is sleep-disordered breathing in patients with idiopathic pulmonary fibrosis? Sleep Breath 14:387-390. https://doi.org/10.1007/ s11325-010-0336-5

Publisher's note Springer Nature remains neutral with regard to jurisdictional claims in published maps and institutional affiliations. 


\section{Authors and Affiliations}

Yuichiro Yasuda $^{1} \cdot$ Tatsuya Nagano $^{1}$ (D) - Shintaro Izumi ${ }^{2} \cdot$ Mina Yasuda $^{3} \cdot$ Kosuke Tsuruno $^{3} \cdot$ Kazunori Tobino $^{3}$. Kyosuke Nakata ${ }^{1,4} \cdot$ Kayoko Okamura $^{5} \cdot$ Teruaki Nishiuma $^{6} \cdot$ Kiyonobu Takatsuki $^{7} \cdot$ Yasuhiro Funada $^{8}$. Hisashi Ohnishi ${ }^{5} \cdot$ Masatsugu Yamamoto $^{1} \cdot$ Yoshihiro Nishimura $^{1} \cdot$ Kazuyuki Kobayashi $^{1}$

Yuichiro Yasuda

yyasuda@med.kobe-u.ac.jp

Shintaro Izumi

shin@cs28.cs.kobe-u.ac.jp

Mina Yasuda

mina0628mina@gmail.com

Kosuke Tsuruno

2ruk0u@gmail.com

Kazunori Tobino

tobino@juntendo.ac.jp

Kyosuke Nakata

nakaticleader@yahoo.co.jp

Kayoko Okamura

k.okamura@amc1.jp

Teruaki Nishiuma

nishiuma@amc1.jp

Kiyonobu Takatsuki

kiyonobu_takatsuki@kitahari-mc.jp

Yasuhiro Funada

funada@ajk.takatsuki-hp.or.jp

Hisashi Ohnishi

ohnishi@amc1.jp

Masatsugu Yamamoto

myamamot@med.kobe-u.ac.jp

Yoshihiro Nishimura

nishiy@med.kobe-u.ac.jp
Kazuyuki Kobayashi

kkoba@med.kobe-u.ac.jp

1 Department of Internal Medicine, Division of Respiratory Medicine, Kobe University Graduate School of Medicine, 7-5-1 Kusunoki-cho, Chuo-ku, Kobe, Hyogo 650-0017, Japan

2 Graduate School of System Informatics, Kobe University, 1-1-Rokkodai-cho, Nada-ku, Kobe, Hyogo 657-8501, Japan

3 Department of Respiratory Medicine, Iizuka Hospital, 3-83 Yoshiomachi, Iizuka, Fukuoka 820-0018, Japan

4 Department of Respiratory Medicine, Kasai City Hospital, 1-13 Yoko, Hojo-cho, Kasai, Hyogo 675-2311, Japan

5 Department of Respiratory Medicine, Akashi Medical Center, 743-33 Yagi, Okubo-cho, Akashi, Hyogo 674-0063, Japan

6 Department of Respiratory Medicine, Kakogawa Central City Hospital, 439 Honmachi, Kakogawa-cho, Kakogawa, Hyogo 675-8611, Japan

7 Department of Respiratory Medicine, Kitaharima Medical Center, 926-250 Ichiba-cho, Ono, Hyogo 675-1392, Japan

8 Department of Respiratory Disease, Takatsuki General Hospital, 1-3-13 Kosobe-cho, Takatsuki, Osaka 569-1192, Japan 